\title{
Dip Solungaç Ağlarında Afalina (Tursiops truncatus) Yunuslar İçin Kullanılan Yunus Kovucu Cihazların İncelenmesi ve Ekonomik Performansı*
}

\author{
Sedat GÖNENER**: UD̆ Ŭ ÖZSANDIKÇI \\ Sinop Üniv., Su Ürünleri Fak., Avlama ve İşleme Teknolojisi Bölümü, Sinop, Türkiye \\ (**Sorumlu yazar e-mail: sedatgonener@gmail.com) \\ DOI: 10.17097/ataunizfd.433660 \\ Geliş Tarihi (Received Date): 13.06.2018 Kabul Tarihi (Accepted Date): 14.01.2019
}

\begin{abstract}
ÖZ: Bu çalışma Sinop liman bölgesinde Eylül 2015 ve Ocak 2017 periyodu içerisinde her ay cihaz kullanan ve kullanmayan balıkçılardan veriler alınarak, dip uzatma ağlarına yunus kovucu cihazlar (pinger) takılmasının akılcı bir uygulama olup olmadığını belirlemek amacıyla yapılmıștır. Balıkçıların kullandıkları fanyasız dip uzatma ağlarının göz açıklığı 34 mm, uzunlukları 1,1 km'dir. Cihazlı ağlarda kullanılan yunus kovucu cihazların frekansı 1-500 kHz olup, etkili mesafeleri 200m'dir. Cihazların şarj edilebilir ve total ömrünün 2-2,5 ay olması ayırt edilebilir özelliğidir. Cihazlı ağlarda (Eylül-Aralık 2015) ilk dört aylık dönemde

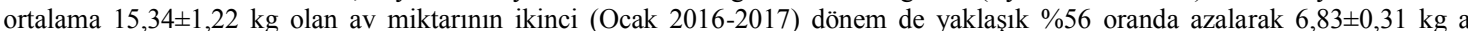
düşmesi çalışmanın önemli bulguları arasında yer almaktadır. Her iki ağ grubuna ait diğger bulgulara göre birim çabada av miktarı cihazlı ağlarda $0,67 \pm 0,02 \mathrm{kgkms}-1$, kontrol ağlarda ise $0,63 \pm 0,02 \mathrm{kgkms}-1$ olarak gerçekleşmiştir. Avlanan balıklardan elde edilen toplam gelir cihazlı ağlarda 22.259,65 TL, kontrol ağlarda ise 20.880,90 TL olarak hesaplanmıştır. Ağlardaki hasar-donatım durumuna ve özellikle ( 2 adetx1500 TL) yunus kovucu cihaz alımına bağlı olarak değişiklik gösteren giderler; cihazlı ağlar için 6400 TL'na ulaşırken, kontrol ağlar için 4000 TL olarak hesaplanmıștır. Buna göre avlama yapılan periyodda cihazlı ağlardan elde edilen net gelirin 15.859,65 TL olduğu hesaplanırken, kontrol ağlardan elde edilen net gelirin bundan \%6 daha fazla gerçekleşerek 16.879,90 TL olduğu belirlenmiştir. Sonuç olarak çalışmada kullanılan yunus kovucu cihazların bölgedeki balıkçı tarafindan kullanılmasının önerilmesi mümkün görünmemektedir.
\end{abstract}

Anahtar Kelimeler: Karadeniz, Dip solungaç ağı, Yunus kovucu cihaz, Etkileşim, Ekonomik performans

\section{Investigation and Economic Performance of Acoustic Deterrent Devices Used in Bottom Gillnets (Pingers) for Bottlenose Dolphin (Tursiops truncatus)}

\begin{abstract}
The study was performed in Sinop between September 2015-January 2017 to investigate whether it is a profitable application to attach pinger devices to bottom-gillnets used in coastal fisheries. Gear specifications of both fishermen group were same as follows; mesh size: $34 \mathrm{~mm}$, length: app. 1,1 km. The frequency range of the pinger device is $1-500 \mathrm{kHz}$ and effective up to $200 \mathrm{~m}$. The most important distinctive feature of those devices is having a built-in rechargeable 1,6 Ah NiMH battery pack but limited up to 2-2,5 months (1000-1500h) total lifetime. In experimental group; average fish catch in the first periods (SeptemberDecember 2015) was $15,34 \pm 1,22 \mathrm{~kg}$ and it decreased by $56 \%$ to $6,83 \pm 0,31 \mathrm{~kg}$ between January 2016-2017. According to results obtained from both control and experimental group, Catch Per Unit Effort (CPUE) was calculated as $0,63 \pm 0,02 \mathrm{kgkms}-1$ and $0,67 \pm 0,02 \mathrm{kgkms}-1$, respectively. Total incomes from the sale of fish were 20880,90 TL and 22259,65 TL for control and experimental groups. The fishermen in control group spent $4000 \mathrm{TL}$ to repair nets damaged due to dolphin presence while other fishermen spent $3400 \mathrm{TL}$ to repair nets. Considering the device expenses for the experimental group (2x1500 TL), total cost was calculated as $6400 \mathrm{TL}$ for experimental group and $4000 \mathrm{TL}$ for control group. Therefore, net income was calculated as 16879,90 $\mathrm{TL}$ in control group while it decreased to $15859,65 \mathrm{TL}$ in experimental group, representing $6 \%$ decline.
\end{abstract}

Keywords: Black Sea, Bottom gillnet, Pinger, Interaction, Economic performance

\section{GíRIŞ}

Yunusların, balık stoklarının azalmasında önemli etkilerinin olduğu, sayıları, tükettikleri balık miktarları, ağlara ve ağlardaki avlanmış balıklara verdikleri zararlar çeşitli çalışmalarla ortaya konmuştur (Öztürk, 1999; Öztürk vd. 1999; Dede, 1999; Birkun, 2002 ve Tonay ve Öztürk 2003). Karadeniz'de ayrıca dünyanın birçok yerinde (Ayadi vd., 2013; Waples vd., 2013; Maccarrone et al., 2014) olduğu gibi 812/2004 sayılı Avrupa Birliği konsey tüzüğü̈-önlemleri ve balıkçıların yunuslarla etkileşimi yönündeki ilgili iddia ve israrlı taleplerle eş zamanlı olarak (rekabeti) tanımlayıcı çalışmalar da yapılmıştır (Gönener ve Bilgin 2007; Gönener ve Bilgin 2009; Gönener ve Özdemir, 2012).

Dünyanın birçok yerinde meydana gelen bu etkileşimler iki başlıkta incelenebilir. Bunlardan ilki ekolojik (dolaylı) rekabettir ve balıkçılarla yunuslar arasında aynı besin kaynakları ve balık stokları için yapılan mücadeleyi ifade etmektedir. Diğeri ise Karadeniz'de özellikle mutur (Phocoena phocoena) yunusların daha çok ip kalınlığı ve göz açıklığı yüksek olan kalkan ağlarına takılarak hedef dışı (bycatch) av olmasi ve afalina (Tursiops truncatus) yunusların dip solungaç ağlarında yakalanmış balıklarla beslenirken ağa zarar vermeleri (depredation) şeklinde oluşan operasyonel rekabettir. Burada afalina yunuslar tarafindan ağlardaki balıkların çalınarak av miktarının azaltılması, bu sırada ağlara verilen zarar ve mutur yunusların ağlara takılıp ölmeleri gibi balıkçılık ekonomisi ve yunusların korunması bakımından negatif sonuçlar ortaya çıkabilmektedir (Lauriano et al., 2004; Cox et al., 2003; CIESM, 2004).

Gerek yunusların korunmas1 gerekse balıkçıların mağduriyetinin giderilmesi için yunusların balık ağlarından uzak tutulması 
gerekmektedir (Birkun, 2002; Birkun vd., 2006). Bu amaçla dünyanın birçok ülkesinde balıç̧ılara, ağlarında sesli caydırıcı cihazlar kullanma zorunluluğu getirilmiştir EUCR (2004).

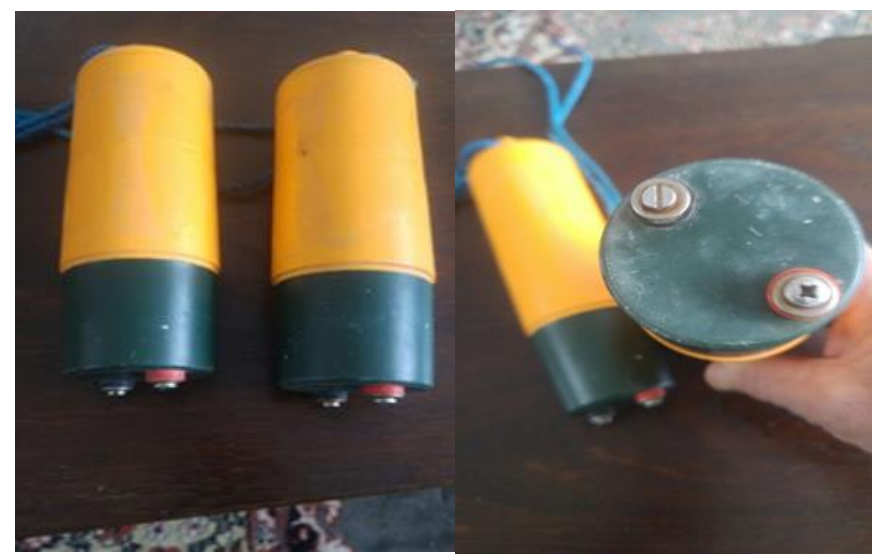

Şekil 1. Çok frekanslı yunus kovucu cihazlar (orijinal)

$\mathrm{Bu}$ çalışmada amaç yunuslarla rekabet halinde olan balıkçıların ekonomik kayıplarının belirlenerek söz konusu etkileşimin azaltılması için kullanılan yunus kovucu cihazların negatif ya da pozitif etkisinin belirlenmesidir. Çalışmada elde edilen bilgi ve bulgular, Karadeniz şartlarında meydana gelen ekolojik rekabetin azaltılması için kimi AB üyesi ülkelerde olduğu gibi alınması gereken önlemlerin değerlendirilmesi (EUCR, 2004) ve özellikle balıkçılar için ortaya çıkan parasal kayıplarla ilgili bilgi boşluklarının doldurulması bakımından güncel, özgün ve önemlidir.

\section{MATERYAL VE METOT}

Çalışma kapsamında Eylül 2015-Ocak 2017 dönemi içerisinde dip solungaç ağlarında yunus kovucu kullanan ve kullanmayan birer balıkçıdan her ay üçer kez olmak üzere avcılığa ait bilgiler alınmıştır. Balıkçılarla yapılan görüşmeler sonucu, avcılık yapılabilen gün sayısının ayda ortalama 20 gün olduğu belirlenmiştir. Her iki balıkçının kullandıkları dip uzatma solungaç ağlarının yapısal ve operasyonal özellikleri aynıdır. Galsama ağ olarak adlandırılan bu fanyasız ağlar multifilament yapıda olup, ağ göz açıklı̆̆ $34 \mathrm{~mm}$, derinlikleri ise (73 göz) 1.3-1.5 m dir. Bölge genelinde balıç̧ların günlük kullandığ1 ağ uzunluğu 1100 m'dir. A ̆g özellikleri bölge genelinde ve verileri araştırmada kullanılan balıkçılardan elde edilmiştir. Balıç̧ılar tarafından kullanılan yunus kovucu cihazlar 1-500 kHz yüksek harmonik (rastgele) frekanslıdır (Şekil 1). Diğerlerinden farklı olarak bu cihazlar ağın kurşun yakasına bağlanmaktadır. Kullanılabilecekleri maksimum derinlik $200 \mathrm{~m}$, cihaz tarafindan üretilen ses dalgalarının etkili mesafesi yani cihaz merkezli yarıçap $200 \mathrm{~m}$ dir. Diğer bir ifade ile ăg kurşun yakasındaki cihazlar arası mesafe 300-400 m olmalı ağa takılacak cihaz sayısı buna göre belirlenmelidir.
$\mathrm{Bu}$ çalışmada kullanılan $1.1 \mathrm{~km}$ lik ağa dört adet cihaz takılması gerekirken, ağın iki ucunu sabitleyen ayak taşlarına (şamandıra ipine ) birer tane olmak üzere iki adet cihaz kullanılmış, ağın kurşun yakasında diğer kısımlara cihaz bağlanmamıştır. $\mathrm{Bu}$ cihazları diğer cihazlardan ayıran bir diğer ve en önemli özellik ise dahili şarj edilebilir 1,6 Ah NiMH pil paketi içermesi ve cihazların toplam kullanım ömrünün (1000-1500 saat) maksimum 2-2,5 ay olmasidir.

Cihazlı ve kontrol ağlarda birim çabada av miktarının belirlenmesi için; [1] Burke (2004) ve Lifelinda, (2007), bu ağlardan elde edilen kazancın hesaplanması için; [2] Buscaino et al., (2009) ve Lifelinda, (2007), her bir teknenin ortalama zararının belirlenmesi için ise; [3] Lauriano et al. (2004) ve Gazo et al., (2008)'in önerdiği formüller kullanılmıştır.

CPUE = C/ST.NL [1]; Burada CPUE: birim çabada av miktarı $\left(\mathrm{kgkms}^{-1}\right), C$ : av miktarı $(\mathrm{kg}), S T$ : ağın denizde kaldığ 1 süre (saat), $N L$ : kullanılan ağ uzunluğudur (km).

$X=$ cpue.kgp, $Y=$ cpue.kgp, $Z=($ cpuex.kgp cpuey.kgp) [2]; $X$ : cihazlı ağlarla birim çabada elde edilen kazanç (TL), cpue: birim çabada av miktarı (kgkms-1), kgp: avlanan türün balıkçı tarafından aracıya satış fiyatı $\left(\mathrm{TLkg}^{-1}\right), Y$ : kontrol ağlarla birim çabada elde edilen kazanç (TL), Z: Birim çabada elde edilen kar (TL/ $\left.\mathrm{kms}^{-1}\right)$ 'dır.

$E D=L . l \cdot f . d \cdot p$ [3]; Burada $E D=$ her bir teknedeki ekonomik zarar (TL), $L$ : ağdaki ortalama av kaybı $\left(\mathrm{kgkm}^{-1}\right), l$ : balıkçılar tarafindan kullanılan günlük ortalama ağ uzunluğu $(\mathrm{km}), d$ : ortalama avlama periyodu (340 gün); $f$ :yunuslarla etkileşim sıklığı ve $p$ : avlanan türün balıkçı tarafından satış fiyatları $\left(\mathrm{TLkg}^{-1}\right)$ ' dır.

Hesaplamalarda avlanan türün balıkçı tarafından aracıya, kooperatife veya kabzımala satış 
fiyatları (TLkg-1) olup, barbunya için 15,0 TLkg-1, mezgit için 8,0 TLkg-1, istavrit için 8,5 TLkg-1, izmarit için 5,0 TLkg-1, çinakop-lüfer için 10,0 TLkg-1 olarak belirlenmiş ve değerlendirme yapılmıştır. Yunuslar tarafından ağlara verilen hasarlar balıkçılar tarafindan 1-1,5 boy (140-210 m) ağın değiştirilmesi şeklinde giderilmektedir. Bir boy ağın yapım maliyeti ağ, yakalar, mantar, kurşun, donatım yaklaşık 400 TL'dır. Çalışma periyodunda 1 $€$ yaklaşık 3,02 TL'dır.

Çalışmada cihazların performansı ortalama av miktarındaki değişim olarak incelendiği için cihazlı ağlarda av miktarının yüksek olduğu periyod I. dönem (Eylül-Aralık 2015), düşmeye başladığı periyod ise II. dönem (Ocak 2016-2017) olarak adlandırılmış, karşılaştırma bu iki dönem arasında yapılmıştır.

Her iki ağ ile avlanan balıkların av miktarları, birim çabada av miktarları ve elde edilen parasal kazanç miktarları parametrik olmayan MannWhitney U test ile karşılaştırılmıştır. Normalite testi olarak Anderson-Darling testi uygulanmış, farklar arasındaki önem derecesi $\mathrm{P}<0,05$ olarak değerlendirilmiştir (Sümbüloğlu ve Sümbüloğlu 2017). Yapilan grafik ve hesaplamalarda MS-Excel ve Minitab 17 programları kullanılmıştır.

\section{BULGULAR}

\section{Cihazların av miktarı ve birim çabada av miktarına etkisi}

Çalışma kapsamında elde edilen toplam 4368,85 kg'lık avın \%51,54'ü cihazlı ağlarla avlanan balıklardan oluşmaktadır. Cihazlı ağlarla ortalama $8,83 \pm 0,44 \mathrm{~kg}$ (Şekil 2), toplam 2251,90 kg, kontrol ağlarla ortalama $8,30 \pm 0,39 \mathrm{~kg}$ (Şekil 3), toplam $2116,95 \mathrm{~kg}$ balık avlanmıştır. Her iki ağla avlanan balık miktarları arasında istatiksel açıdan herhangi bir fark yoktur $(\mathrm{P}=0,481)$.

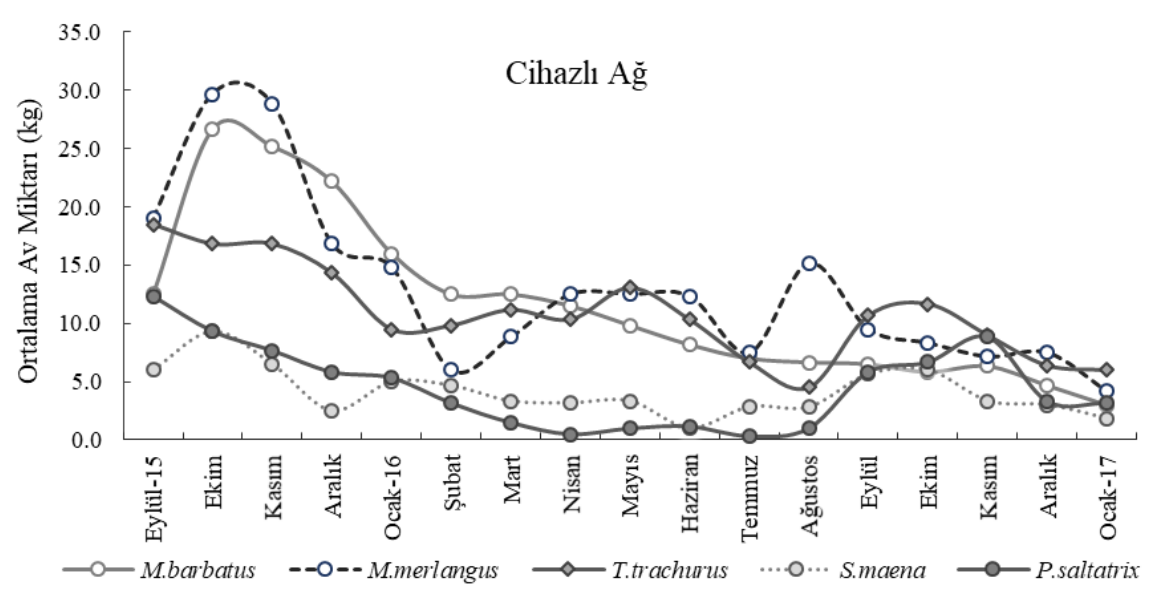

Şekil 2. Cihazlı ağlarda türlere göre aylık ortalama av miktarı

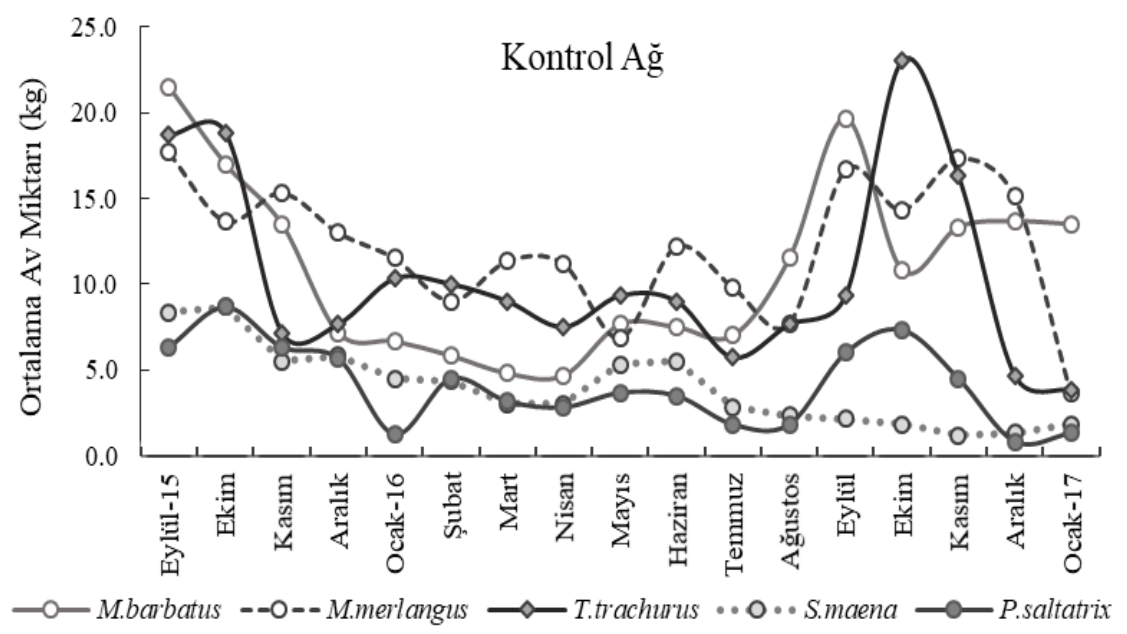

Şekil 3. Kontrol ağlarda türlere göre aylık ortalama av miktarı 
Cihazlı ve kontrol ağlarda birim çabada av miktarlar1 sirasiyla $0,67 \pm 0,03 \mathrm{~kg} / \mathrm{kms}^{-1}$ ve $0,63 \pm 0,03$ $\mathrm{kg} / \mathrm{kms}^{-1}$ 'dır. Her iki ağın birim çabada av miktarları arasında önemli bir fark yoktur.

Cihazlı ağlarda (Eylül-Aralık 2015) ilk dört aylık dönemde ortalama $15,34 \pm 1,22 \mathrm{~kg}$ olan av miktarı ikinci (Ocak 2016-2017) dönem \%56 azalarak 6,83 $\pm 0,31 \mathrm{~kg}$ 'a düşmüştür. Birim çabada av miktarları I. ve II. dönemler için sırasıyla 1,16 $\pm 0,09$ $\mathrm{kg} / \mathrm{kms}^{-1}$ ve $0,86 \pm 0,06 \mathrm{~kg} / \mathrm{kms}^{-1}$ olarak belirlenmiş ve aralarındaki fark istatistiksel bakımdan önemli bulunmuştur ( $\mathrm{P}=0,021)$.

Dip uzatma solungaç ağlarında avcıllı̆g hedeflenen türler içerisinde ilk sıralarda yeralan barbunya (M. barbatus) ve mezgit (M. merlangus euxinus) balıklarının birim çabada av miktarlarının cihazlı ağlarda daha fazla olduğu belirlenmiş ve kontrol ağlarla aralarındaki fark önemli bulunmuştur ( $\mathrm{P}=0,013$ ve $\mathrm{P}=0,032$ ) (Çizelge 1).

Çizelge 1. Birim çabada av miktarlarının türlere göre değişimi $\left(\mathrm{kg} / \mathrm{kms}^{-1}\right)$

\begin{tabular}{|c|c|c|c|}
\hline Avlanan Türler & Cihazlı Ăg & Kontrol Ă & $\mathbf{P}$ \\
\hline M. barbatus & $1,27 \pm 0,09$ & $0,92 \pm 0,07$ & 0,013 \\
\hline M. merlangus euxinus & $0,65 \pm 0,05$ & $0,50 \pm 0,04$ & 0,032 \\
\hline T. mediterraneus & $0,53 \pm 0,05$ & $0,49 \pm 0,03$ & 0,705 \\
\hline S. smaris & $0,50 \pm 0,04$ & $0,68 \pm 0,07$ & 0,179 \\
\hline$P$. saltatrix & $0,40 \pm 0,02$ & $0,54 \pm 0,07$ & 0,740 \\
\hline Genel & $0,67 \pm 0,03$ & $0,63 \pm 0,03$ & 0,481 \\
\hline
\end{tabular}

İki ağ grubunda avlanmış diğer türler ve genel olarak birim çabada av miktarları arasındaki farklar ise önemsizdir $(\mathrm{P}=0,481)$.

\section{Ekonomik performans}

Avlama dönemi boyunca türlerin toplam av miktarları esas alınarak hesap yapıldığında elde edilen gelirin cihazlı ağlarda 22259,65 TL, kontrol ağlarda ise 20880,00 TL olduğu belirlenmiştir. Avcilık sırasinda afalina yunuslar tarafindan yırtılan, avc1lık yapamayacak düzeyde hasar gören ve yeniden donatılan kısımlar kontrol ağlarda 10 boy $(1,1 \mathrm{~km})$, cihazlı ağlarda ise $8,5(0,93 \mathrm{~km})$ boydur. Dolayısıyla bu ağların donatılması için yapılan harcama kontrol ağlar için $4000 \mathrm{TL}$, cihazlı ağlar için ise $3400 \mathrm{TL}$ olarak hesaplanmıştır. Ağlardaki hasar-donatım durumuna ve özellikle ( 2 adet x $1500 \mathrm{TL}$ ) yunus kovucu cihaz alımına bağlı olarak değişiklik gösteren giderler; cihazlı ağlar için 6400 TL'na ulaşırken, kontrol ağlar için 4000 TL olarak hesaplanmıştır. Buna göre avlama yapılan periyodda cihazlı ağlardan elde edilen net gelirin 15859,65 TL olduğu, kontrol ağlardan elde edilen net gelirin ise 16880,00 TL olduğu belirlenmiştir (Çizelge 2).

Çizelge 2. Av sezonu boyunca her iki ağ grubunda gelir gider analizi (TL) (C: Av miktarı, kgp: Kilogram fiyat1)

\begin{tabular}{lccc}
\hline Avlanan Türler & Cihazlı (C.kgp) & Kontrol (C.kgp) & Fark \\
\hline M. barbatus & 8865,00 & 8287,50 & 577,50 \\
M. merlangus euxinus & 5296,00 & 4952,00 & 344,00 \\
T. mediterraneus & 4733,65 & 4540,70 & 192,95 \\
S. smaris & 1055,00 & 1012,50 & 42,50 \\
$P$. saltatrix & 2310,00 & 2087,50 & 222,50 \\
Gelir toplam & 22259,65 & 20880,00 & 1379,75 \\
Ağ donatım & 3400,00 & 4000,00 & - \\
Yunus kovucu cihaz & 3000,00 & - & - \\
Gider toplam & 6400,00 & 4000,00 & - \\
\hline Kalan & 15859,65 & 16880,00 & 1020,35 \\
\hline
\end{tabular}

Çalışma kapsamında bölgede benzer şekilde ve benzer materyalle avcılık yapan her bir teknenin afalinalar tarafından gerçekleştirilen (depredation) yağmalama, ağda avlanmış haldeki balıklardan beslenme faaliyeti nedeniyle oluşabilecek zararları 
3028,28 TL $(\approx 1002.74 €)$ olarak hesaplanmıştır (Çizelge 3).

Birim çabada avlanan balıklardan elde edilen kazanç Çizelge 4'de verilmiş olup, cihazlı ağlarda
$35,21 \mathrm{TL} / \mathrm{kms}^{-1}$, kontrol ağlarda ise $30,90 \mathrm{TL} / \mathrm{kms}^{-}$

${ }^{1}$ 'dir. Buna göre cihazlı ağlarda birim çabada 4,31 $\mathrm{TL} / \mathrm{kms}^{-1}$ daha fazla kazanç elde edilmiştir

Çizelge 3. Yunuslardan kaynaklanan tekne başına ekonomik zarar ( $E D=$ her bir teknedeki ekonomik zarar (TL), L: ağdaki ortalama av kaybı $\left(\mathrm{kgkm}^{-1}\right), l$ : balıkçılar tarafindan kullanılan günlük ortalama ağ uzunluğu $(\mathrm{km}), d$ : ortalama avlama periyodu; $f$ :yunuslarla etkileşim sıklığı, $p$ : avlanan türün balıkçı tarafından satış fiyatları $\left.\left(\mathrm{TLkg}^{-1}\right)\right)$

\begin{tabular}{|c|c|c|c|c|c|c|}
\hline Türler & $f$ & $d$ (gün) & $l(\mathrm{~km})$ & $L(\mathrm{~kg} / \mathrm{km})$ & $p(k g / T L)$ & $E D(T L)$ \\
\hline M. barbatus & 0,04 & 340 & 1,1 & 35,0 & 15,0 & 7854,00 \\
\hline M. m. еuxinus & & & & 39,0 & 8,0 & 4667,52 \\
\hline T. mediterraneus & & & & 20,2 & 8,5 & 2568,63 \\
\hline S. smaris & & & & 0,77 & 5,0 & 57,60 \\
\hline P. saltatrix & & & & 20,2 & 10,0 & 3021,92 \\
\hline Ortalama Ekonomik Zarar & & & & 3028,28 & & \\
\hline
\end{tabular}

Çizelge 4. Cihazlı ve Kontrol ağlarda birim çabada av miktarından (cpue) elde edilen kazanç (TL/kms $\left.{ }^{-1}\right)(X$ : cihazlı ağlarla birim çabada elde edilen kazanç (TL), $Y$ : kontrol ağlarla birim çabada elde edilen kazanç (TL), Z: Birim çabada elde edilen $\operatorname{kar}\left(\mathrm{TL} / \mathrm{kms}^{-1}\right)$, kgp: Kilogram fiyatı (TL))

\begin{tabular}{lccc}
\hline Avlanan Türler & X (cpue.kgp) & Y (cpue.kgp) & Z (cpuex.kgp-cpuey.kgp) \\
\hline M. barbatus & 19,0 & 13,86 & 5,14 \\
M. m. euxinus & 5,18 & 4,01 & 1,17 \\
T. mediterraneus & 4,52 & 4,20 & 0,32 \\
S. smaris & 2,48 & 3,41 & $-0,93$ \\
$P$. saltatrix & 4,03 & 5,42 & $-1,39$ \\
\hline Toplam & 35,21 & 30,90 & 4,31 \\
\hline
\end{tabular}

TARTIŞMA VE SONUÇ

Yapılan çalışmada Eylül 2015-Ocak 2017 tarihleri arasındaki 17 aylık dönemde yunus kovucu cihaz bulunan (cihazlı) ve bulunmayan (kontrol), aynı teknik özelliklere sahip iki dip uzatma ağının türlere göre aylık av miktarları alınmıştır. Çalışma kapsamında her iki ağ için ortalama av miktarları, birim çabadaki av miktarları her bir teknenin yunus kaynaklı ekonomik zararları ve birim çabadaki kazançları belirlenmiştir.

Birim çabada av miktarları karşılaştırıldı ğında, mezgit ve barbunya balıklarının cihazlı ağlarda daha fazla avlandığı görülmektedir. Bu durum, cihazların etkili oldukları düşünülen ilk aylarda yunusları ağdan uzak tutarak, hedef türler olan mezgit ve barbunya balıklarının av miktarını arttırdığı ve aradaki farkın bundan kaynaklandığı şeklinde açıklanabilir.

Diğer balıkçıların cihaz bulunduğu bilinen ağa dikine veya paralel ancak 1srarla yakın (kardeş) ağlar atmalarından başka maliyetinin yüksek olması nedeniyle balıkçı tarafından sadece iki adet cihaz kullanılmış olması dolayısıyla ortamda yunuslar için geçiş boşluklarının oluşabileceği gibi durumların cihazlı ağlarda av kayıplarına ve av miktarlarında bazı değişikliklere neden olabileceği değerlendirilmelidir.

Yunus kovucu cihazların etkisi çok sayıda araştırmacı tarafından ekonomik açıdan incelenmiştir (Çizelge 5). Akdeniz, Karadeniz ve Atlantik’te yapılan çalışmalar, kullanılan tek sinyalli cihazların bir ikaz veya uyarı ekipmanı olarak mutur yunusların dip solungaç ağlarına takılmasını önlemede başarılı olduğunu göstermektedir (Gazo et al., 2001; Gönener ve Bilgin 2007, 2009; Cox et al., 2003). Ancak, Orphanides and Palka (2013) tarafindan kuzeybatı Atlantik'te beş bölgede on iki yıl süre ile yapılan bir çalışmada hedef dışı avın beklenen seviyede azaltılamayacağı bildirilmiştir. Tek sinyalli yunus kovucu cihazların başarısının sınırlı olmasının yanısıra son derece firsatçı (opportunistic) beslenme alışkanlıklarına sahip afalina yunusların dip solungaç ağlarından uzaklaştırılmasında etkili sonuçlar vermeyebileceği bildirilmektedir (Burke, 2004; Lauriano et al., 2004). Bu nedenle çoklu frekansa sahip, yunus kovucu cihazlar üretilmiş ve bu cihazların etkisi de birçok çalışmada araştırılmıştır (Şekil 1). Gönener ve Bilgin (2007), Gönener ve Özdemir (2012), Brotons et al. (2008a), Gazo and 
Aguilar (2002), Gazo et al. (2008), Buscaino et al. (2009), Burke (2004), Lopez and Marino (2011) tarafindan yapılan bazı çalışmalarda yunus kovucu cihazların afalina yunuslar üzerinde çeşitli düzeylerde başarılı veya başarısız olduğu belirlenmiş̧tir. Diğer araştırmalardan çok daha farklı olarak Orta Akdeniz'de Kerkennah Adaları çevresinde çalışma yapan Ayadi et al. (2013) beklenenin aksine yunus kovucu cihazların yunusları ağa çektiğini ve genel olarak yunus kovucu cihaz yerleştirilen ağların kontrol ağlara göre daha fazla saldırıya uğradığını ifade etmiştir. Ayrıca bu çalışmada kullanılan yunus kovucu cihazların avcılığı hedeflenen mürekkepbalığı (Sepia officinalis) türlerini de negatif etkilediği ve birim çabada av miktarını \%22 oranda azalttığı bildirilmiştir.

Çizelge 5. Yunusların neden olduğu ekonomik zararlara $(€)$ ilişkin farklı çalışmalara ait sonuçlar $(E D=$ her bir teknedeki ekonomik zarar (TL)).

\begin{tabular}{lll}
\hline Yazar & Bölge & ED (€) \\
\hline Cruz et al. (2014) & Portekiz-Azor & 423,73 \\
Lauriano et al. (2004) & Italya & 1168,24 \\
Gazo et al. (2008) & İspanya-Mayorka & 1094,0 \\
Gönener ve Özdemir (2012) & Türkiye-Sinop & 1052,03 \\
Maccarone et al. (2014) & Italya-Sicilya & 1400,0 \\
\hline Bu çalış̧a (2015-2017) & Türkiye-Sinop & 1002,74 \\
\hline
\end{tabular}

Her bir teknede yunuslardan kaynaklı ekonomik zararın 3028.28TL $\quad \approx 1002,74 \quad €)$ olduğu belirlenmiştir. $\mathrm{Bu}$ finansal kayıp yapılan diğer çalışmalarda elde edilen sonuçların birçoğu ile benzerlik göstermektedir. Çalışmada kullanılan cihazlı ağdaki iki adet yunus kovucu cihazın maliyetinin $3000 \mathrm{TL}$ olduğu göz önüne alındığında, cihazın sağlayacağı yararın zaten ortadan kalktığı anlaşılmaktadır.

Mevcut çalışmada balıkçıların kullandığı ve yararlılığ 1 incelenen şarjlı yunus kovucu cihazlar, Maccarone et al. (2014) in kullandığı cihazlarla aynı teknik özellikleri taşımaktadır. Sinop bölgesinde 2015 y1linda tanesi 200-223 € olmasi gereken (Maccarone et al. 2014; Fortuna and Northridge, 2005) bu cihazlar balıkçılarımız tarafından tanesi yaklaşık 1500 TL'na $(\approx 450 \quad €)$ satın alınarak kullanılmıştır. Sicilya-Egadi adası yakınlarında yaptığı çalışmada Maccarone et al. (2014) AB Balıkçılık Fonu (EFF) yardımıyla dört cihazı $800 €$ olmak üzere alarak kullanmış, buna rağmen mevcut çalışmada olduğu gibi cihazın maliyetini yüksek, performansını ise düşük bulmuştur.

Balıkçıların ağlarda cihazların kullanılıp, kullanılmaması kararını en çok etkileyecek bulgulardan birisi de birim çabada av miktarı karşılığı elde edilecek parasal kazançtır. Bu model cihazların şarj edilebilir olması iyi bir özellik olmasına rağmen toplam kullanım ömürlerinin de 1000-1500 saat yani 2-2.5 ay ile sinırlı olması önemli bir teknik bilgidir. Çünkü bu kullanım süresinin sonunda yani çalışmadığ (yunuslar tarafindan tanınarak ağa, tekneye yönelmeleri gibi) beklenenin tersine etkiler yapabilmesi de mümkündür. Ancak bu durum fiyatları 2-3 kat pahalı da olsa cihazı satın alan balıkçılar tarafından bilinmemekte, birbirini tekrar eden avcılık faaliyetleri sırasında sahada kısmen öğrenilmektedir.

Çalışma bulgularına bakıldığında cihazlı ağlarda hedef türler olan barbunya ve mezgit türlerinin birim çabada daha fazla kazandırdığı görülmektedir. Ancak bir buçuk yıldan fazla süren bu çalışma sonunda cihazları alıp, kullanan balıkçıların cihaz kullanmayan balıkçılara göre 1020,35 TL zarar etmeleri çalışmanın önemli bulguları arasındadır (Çizelge 2).

Cihazların; yunusları habitat dışına kovma, yunuslarda duyma bozuklukları, onların ekolokasyonlarla ilerlemesini de engelleyecek şekilde ortamda gürültü kirliliği, kanıksama, alışma, yemek zili olarak çă̆ırma ve hatta sardalya, ringa, hamsi gibi duyma frekansı çok düşük olan balık türlerinin av oranında azalma gibi yan etkileri olabileceği bazı önemli çalışmalarda belirtilmektedir (Bearzi et al., 2008; Brotons et al., 2008b; Dawson et al., 1998; Zahri et al., 2004). Mevcut çalışma sonuçları ise bu cihazlara yapılan yatırım maliyetinin çok yüksek olduğunu ve bu maliyetin geri dönüş süresininin ekonomik analiz sonuçlarını değiştiren en önemli ve en kritik faktör olduğunu göstermektedir. Kullanım süresinin 2-2,5 ay olduğu göz önüne alındığında cihazın; maliyetinin yüksek ve geri dönüş süresinin çok uzun olması hatta kendini amorti etmesinin pek mümkün olmaması gibi nedenler kullanımının ekonomik ve doğru bir yaklaşım olmayacağını göstermektedir. 


\section{KAYNAKLAR}

Ayadi, A., Mohamed, G., Bradai, M.N., 2013. Do pingers reduce interactions between bottlenose dolphins and trammel nets around the Kerkennah Islands (Central Mediterranean Sea). Cahiers de Biologie Marine, 54(3): 375-383.

Bearzi, G., Fortuna, C.M., Randall, R.R., 2008. Ecology and conservation of common bottlenose dolphins Tursiops truncatus in the Mediterranean Sea. Mammal Review, 39 (2): 92-123. doi: 10.1111/j.13652907.2008.00133.x

Birkun, A. Jr., 2002. Interactions between cetaceans and fisheries in the Black Sea. In: G. Notarbartolo di Sciara (Ed.), Cetaceans of the Mediterranean and Black Seas: state of knowledge and conservation strategies. A report to the ACCOBAMS Secretariat, Monaco.

Birkun, A.Jr., Cañadas, A., Donovan G., Holcer, D., Lauriano, G., Notarbartolo di Sciara, G., Panigada, S., Radu, G., van Klaveren, M.C., 2006. Conservation Plan for Black Sea Cetaceans. Agreement on the Conservation of Cetaceans of the Black Sea, Mediterranean Sea and Contiguous Atlantic Area.

Brotons, J.M., Grau, A. M., Rendell, L., 2008a. Estimating the impact of interactions between bottlenose dolphins and artisanal fisheries around the Balearic Islands. Society for Marine Mammalogy, 24(1): 112-127. doi: 10.1111/j.1748-7692.2007.00164.x

Brotons, J.M., Munilla, Z., Grau, M. A., Rendell, L., 2008b. Do pingers reduce interactions between bottlenose dolphins and nets around the Balearic Islands? Endangered Species Research, 5: 301-308. doi: 10.3354/esr00104

Burke, E.K., 2004. The effect of acoustic deterrent devices on bottlenose dolphin depredation in the Spanish mackerel gillnet fishery. Duke University, Msc Dissertation., Nicholas, USA.

Buscaino, G., Buffa, G., Sarà, G., Bellante, A., Tonello, A.J.J., Hardt, F.A.S., Cremer, M. J., Bonanno, A., Cuttitta, A., Mazzola, S., 2009. Pinger affects fish catch efficiency and damage to bottom gill nets related to bottlenose dolphins. The Japanese Society of Fisheries Science, 75: 537-544.

CIESM, 2004. Investigating the roles of cetaceans in marine ecosystems. CIESM Workshop Monograph no 25, pp. 144, Monaco

Cox, T.M., Read, A.J., Swanner, D., Urian, K., Waples, D., 2003. Behavioral responses of bottlenose dolphins, Tursiops truncatus, to gillnets and acoustic alarms. Biological Conservation, 115: 203-212.

Cruz, M. J., Jordão, V. L., Pereira, J. G., Santos, R. S., Silva, M.A., 2014. Risso's dolphin depredation in the Azorean hand-jig squid fishery: assessing the impacts and evaluating effectiveness of acoustic deterrents. ICES Journal of Marine Science, 71: 2608-2620. doi:10.1093/icesjms/fsu073

Dede, A., 1999. Türk Boğazlar Sisitemi'nde yaşayan deniz memelileri populasyonları üzerine araştırmalar. İstanbul Üniversitesi Fen Bilimleri Enstitüsü, Doktora Tezi, İstanbul.

Dawson, S.M., Read, A.J., Slooten, E., 1998. Pingers, porpoises and power; uncertainties with using pingers to reduce bycatch of small cetaceans. Biological Conservation 84: 141146.

EUCR, 2004. No 812/2004 of 26.4.2004, Laying down measures concerning incidental catches of cetaceans in fisheries and amending Regulation (EC) No 88/98.

Fortuna, C., Northridge, S., 2005. Pingers - A technological update on the issue, with a view of developing common strategies on the competition issue: a pragmatic approach. Agreement on the Conservation of Cetaceans of the Black. Third Meeting of the Scientific Committee. Cairo, 14/17 May 2005.

Gazo, M., Fernandez-Contreras, M., Brotons, J.M., Aguilar, A., 2001. Interactions between bottlenose dolphins and artisanal fisheries in the Balearic Islands: may acoustic devices be a solution to the problem? Eur. Res. Cetaceans [Abstracts] 15: 156-157. Proceedings of the fifteenth annual conference of the European Cetacean Society, Rome, Italy, 6-10 May 2001.

Gazo M., Aguilar, A., 2002. Are pingers useful to reduce damage produced by bottlenose dolphins in trammel nets? a test in the Balearic Islands. Scientific Committee of the International Whaling Commission, Shimonoseki, May 2002, SC/54/SM11. $6 \mathrm{p}$.

Gazo, M., Gonzalvo, J., Aguilar, A., 2008. Pingers as deterrents of bottlenose dolphins interacting with trammel nets. Fisheries Research, 92: 7075.

Gönener, S., Bilgin, S., 2007. Sinop Yarımadası civarında (Karadeniz, Türkiye) dip uzatma galsama ağlarında yunusların balıkları çalmaları üzerine akustik pingerlerin etkisi. Fırat Üniversitesi Fen ve Mühendislik Bilimleri Dergisi, 19(2): 121-127.

Gönener, S., Bilgin, S., 2009. The effect of pingers on harbour porpoise, Phocoena phocoena bycatch and fishing effort in the turbot Gill net fishery in the Turkish Black Sea Coast. Turkish Journal of Fisheries and Aquatic Sciences, 9 (2): 151-158

Gönener, S., Özdemir, S., 2012. Investigation of the interaction between bottom gillnet fishery 
(Sinop, Black sea) and bottlenose dolphins (Tursiops truncatus) in terms of economy. Turkish Journal of Fisheries and Aquatic Sciences, 12: 115-126.

Lauriano, G., Fortuna, C.M., Moltedo, G., Notarbartolo di Sciara, G., 2004. Interactions between common bottlenose dolphins (Tursiops truncatus) and the artisanal fishery in Asinara Island National Park (Sardinia): assessment of catch damage and economic loss. Journal of Cetacean Research and Management, 6(2):165173.

Lifelinda, 2007. Estimation des variations de production dans les filets de peche en cas d'interaction avec le grand dauphin et definition avec les pecheurs professionnels de strategies potentielles d'evitement des dauphins. Life Linda. Office de L'environnement de la Corse: Réserve Naturelle des Bouches de Bonifacio. Rapport Final 15 Avril 2007, Université De Corse, $69 \mathrm{p}$.

López, B.D., Mariño, F., 2011. A trial of acoustic harassment device efficacy on free-ranging bottlenose dolphins in Sardinia, Italy. Marine and Freshwater Behaviour and Physiology, 44: 197-208.

Maccarone, V., Buffa, G., Stefano, V.D., Filiciotto, F., Mazzola, S., Buscaino, G. 2014. Economic assessment of dolphin depredation damages and pinger use in artisanal fisheries in the archipelago of Egadi Islands (Sicily). Turkish Journal of Fisheries and Aquatic Sciences, 14: 173-181.
Orphanides, C.D., Palka, D.L., 2013. Analysis of harbor porpoise gillnet bycatch, compliance, and enforcement trends in the US northwestern Atlantic, January 1999 to May 2010. Endang Species Research, 20: 251-269.

Öztürk, B., 1999. Black Sea Biological Diversity: Turkey. GEF Black Sea Environmental Programme, United National Publications, Newyork, 144 p.

Öztürk, B., Öztürk, A.A., Dede, A., 1999. Cetacean bycatch in the western coast of the Turkish Black Sea in 1993-1997. In: G.H., Evans, J. Cruz, J.A., Raga (Eds.), European research on cetaceans - Proc. 13 ${ }^{\text {th }}$ Annual Conf. European Cetacean Society, Valencia, Spain.

Tonay, A. M., Öztürk, B., 2003. Cetacean bycatches in turbot fishery on the western coast of the Turkish Black Sea. In: Int. Symp. of Fish. and Zool. Istanbul (pp. 131-138).

Sümbüloğlu, K., Sümbüloğlu, V., 2017. Biyoistatistik (18.Bask1). Hatipoğlu Yayınevi, Ankara.

Waples, D.M., Thorne, L.H., Hodge, L.E.W., Burke, E.K., Urian, K.W., Read, A.J. 2013. A field test of acoustic deterrent devices used to reduce interactions between bottlenose dolphins and a coastal gillnet fishery. Biological Conservation, 157:165-171.

Zahri, Y., Abid, N., Elouamari, N., Abdellaoui, B., 2004. Étude de l'interaction entre le grand dauphin et la pêche á la senne coulissante en Méditerranée marocaine. INRH Report, Casablanca. 